\title{
Relation of prediabetes and type 2 diabetes mellitus to thyroid cancer
}

\author{
T Grimmichova ${ }^{1,2}$, M Haluzik ${ }^{1,3}$, K Vondra $^{1}$, P Matucha $^{1}$ and M Hill1 \\ IInstitute of Endocrinology, Narodni, Prague, Czech Republic \\ 22nd Department of Internal Medicine, University Hospital Kralovske Vinohrady and Third Faculty of Medicine, Charles University in Prague, Srobarova, \\ Prague, Czech Republic \\ ${ }^{3}$ Diabetes Centre, Institute for Clinical and Experimental Medicine (IKEM), Videnska, Prague, Czech Republic
}

Correspondence should be addressed to T Grimmichova: tgrimmichova@endo.cz

\begin{abstract}
Objective: Patients with type 2 diabetes (T2DM) generally experience a higher incidence of cancer. However, the association between T2DM and thyroid cancer is inconclusive. Methods: Case-control prospective study, where 722 patients were screened for T2DM and prediabetes (PDM) and underwent thyroid ultrasound and biochemical tests. The patients were assigned to groups of PDM $(n=55)$, T2DM $(n=79)$ or a non-diabetes group (NDM) ( $n=588)$. Fine-needle aspiration biopsy was carried out in 263 patients. Histological examinations were done for 109 patients after surgery, with findings of 52 benign (BS) and 57 malignant tumors (MS).

Results: Thirty-three percent of patients with T2DM and especially PDM were newly diagnosed by our screening: $6.5 \%$ with T2DM and $72 \%$ with PDM, respectively. The percentage of thyroid cancers did not significantly differ between the groups $\left(\chi^{2}\right.$ test $=0.461 ; P=0.794$ ). Relevant positive thyroid predictors for T2DM (t-statistic $=25.87$; $P<0.01)$ and PDM $(21.69 ; P<0.01)$ contrary to NDM $(-26.9 ; P<0.01)$ were thyroid volume $(4.79 ; P<0.01)$, thyroid nodule volume $(3.25 ; P<0.01)$ and multinodular thyroid gland $(4.83 ; P<0.01)$, while negative relevant predictors included the occurrence of autoimmune thyroid disease (AITD) $(-2.01 ; P<0.05)$.

Conclusion: In general, we did not observe an increased risk for thyroid cancer in the diabetic and prediabetic groups in comparison to controls, in spite of well-established increased risk for other malignancies. Structural and benign changes such as larger and multinodular thyroid glands, in comparison to autoimmune thyroid disease, are present more often in diabetics.
\end{abstract}

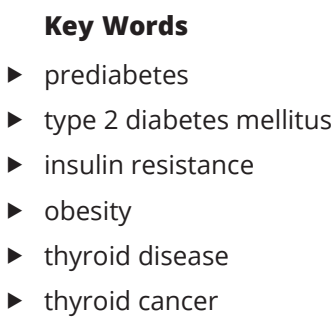

Endocrine Connections (2020) 9, 607-616

\section{Introduction}

Thyroid cancer (TC) is a relatively rare cancer, with an incidence from 1 to $5.3 \%$ of all malignancies, but it represents the most common malignancy originating from the endocrine organs. The most common TCs are differentiated carcinomas, with majority being papillary carcinomas (PTC), especially micropapillary carcinoma (PMTC). TC is more frequent in women than in men and it is currently the third most common cancer in women under the age of 45 in highly developed countries $(1,2,3)$. In spite of its striking increase of incidence in both men and women, the mortality rate for thyroid cancer has not changed and has remained low (women 3\%; men 5\%). Nevertheless, in some studies, mortality is increased in $\geq 40$ years-old age groups, and annual mortality has increased by $1 \%$ in men by $3 \%$ for advanced PTC (1974-2013). An increase of larger TC tumors $(>5 \mathrm{~cm})$ has also been noted $(4,5)$.

TC differs from other tumors in age distribution, with the most-commonly affected group patients in the

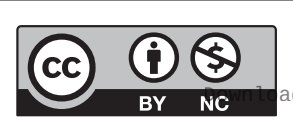


economic productive age of 24-60 years, while in other malignancies the risk of carcinoma increases continuously with age. Therefore, secondary causes of this increase in TC incidence have been suggested. They include, briefly: (1) ionizing radiation, which is dose dependent with the highest sensitivity in childhood; however, less than $5 \%$ of all TC are associated with radiotherapy (6). (2) Environmental factors, including endocrine disrupting chemicals affecting the endocrine system, though there is lack of direct evidence in humans (7). (3) Hormonal changes especially estrogens in the reproductive period of women contribute to the higher TC incidence in women. It should be emphasized that while women are diagnosed with TC more frequently than men, in autopsy studies the female/male ratio is the same (8). (4) Autoimmune thyroid disease (AITD). Studies have produced contradictory results and AITD can even be a protective factor in TC progression $(9,10)$. (5) Both iodine deficiency and overconsumption have been suggested to be risk factors for TC. Obviously, follicular thyroid cancer occurs predominantly in iodinedeficient countries in comparison with PTC in iodinesufficient countries (11). (6) Familial non-medullary thyroid carcinoma, which with a $3-10 \%$ prevalence is more frequent in comparison with other tumors (12). (7) TC incidence may have also been increased due to better TC detection ('overdiagnosis') depending on the numbers of physicians providing ultrasound and ultrasound-guided fine-needle aspiration (6). Finally, and the topic of our study, (8) type 2 diabetes (T2DM), obesity and insulin resistance (IR) in the framework of the worldwide epidemic of diabetes and obesity. In general, patients with diabetes have an approximately $20-25 \%$ higher risk of cancer in comparison with patients without diabetes. Epidemiological studies have consistently reported that individuals who are either overweight or obese are at an increased risk of thyroid cancer, but the results are inconsistent for diabetic patients. Both obesity and type 2 diabetes are characterized by insulin resistance, hyperinsulinemia and the overproduction of other growth factors $(13,14)$. The relationship between T2DM and thyroid cancer is unclear, so in our study we explored whether the presence of diabetes affects the risk of thyroid cancer.

\section{Materials and methods}

The protocol of this study complies with the Declaration of Helsinki, and before entering the study, written informed consent was obtained from patients after they received both written and oral information. The study was approved by the ethical committee of Institute of Endocrinology. In our prospective case-control study, we included randomly 561 women and 161 men in a country with iodine sufficiency (15). The power of the sample size was established. The patients were recruited from October 2013 to February 2018. Patient history, ultrasound of neck (US), and biochemical testing were done at the Institute of Endocrinology and 2nd Department of Internal Medicine, University Hospital Kralovske Vinohrady (a secondary referral center). The patients were recruited into the study during the first appointment at the outpatient clinic (Fig. 1). These patients were recommended to our department from primary care mainly due to suspicions of thyropathy, other endocrinopathy or diabetes. Patients with negative history of prediabetes or diabetes were screened for diabetes following the standards of the American Diabetes Association and the European Association for the Study of Diabetes. Patients with prediabetes are defined by the presence of impaired fasting glucose (IFG) and/or impaired glucose tolerance (IGT) and/or HbA1c $39-47 \mathrm{mmol} / \mathrm{mol}$. IFG is defined as fasting plasma glucose (FPG) levels between 5.6 and $6.9 \mathrm{mmol} / \mathrm{L}$ and IGT as 2-h plasma glucose levels during a $75 \mathrm{~g}$ oral glucose tolerance test (OGTT) between 7.8 and $11.0 \mathrm{mmol} / \mathrm{L}$. Diabetes may be diagnosed based on plasma glucose criteria, either using the FPG value or the 2-h plasma glucose (2-h PG) value during an OGTT, or A1C criteria. Criteria for the diagnosis of diabetes: $\mathrm{FPG} \geq 7.0 \mathrm{mmol} / \mathrm{L}$ or 2 -h $\mathrm{PG} \geq 11.1$ $\mathrm{mmol} / \mathrm{L}$ during OGTT or A1C $\geq 6.5 \%(48 \mathrm{mmol} / \mathrm{mol})$ or in a patient with classic symptoms of hyperglycemia or hyperglycemic crisis, a random plasma glucose $\geq 11.1$ $\mathrm{mmol} / \mathrm{L}(16,17)$. The homeostatic model assessment of insulin resistance (HOMA-IR) was calculated using the following formula: HOMA-IR=(fasting C-peptide $(\mathrm{nmol} / \mathrm{L}) \times$ fasting glucose $(\mathrm{mmol} / \mathrm{L}) / 22.5)$. The HOMA estimates steady state beta-cell function (\%B; HOMA-B) and insulin sensitivity (\%S; HOMA-S), as percentages of a normal reference population (18). Antidiabetic treatment data were not assessed in detail. However, the antidiabetic treatment was as follows: metformin $53 \%$, intensive insulin therapy $15.2 \%$, diet $12.1 \%$, combination of insulin with peroral antidiabetics (PAD) $7.6 \%$, combination of PAD 6.1\%, GLP-1 analogs 3\%, gliptins $1.5 \%$, and sulfonylureas $1.5 \%$ patients.

The diagnosis of autoimmune thyroid disease (AITD) was based on positive thyroid autoantibodies and/or the hypoechogenic pattern typical for AITD during the US examination. Only 28 patients with Graves-Basedow disease were included in the study. Patients with hypo-

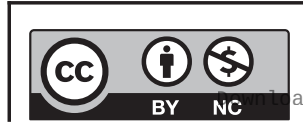

This work is licensed under a Creative Commons Attribution-NonCommercial 4.0 International License. ed from Bioscientifica.com at 04/26/2023 09:51:51AM 


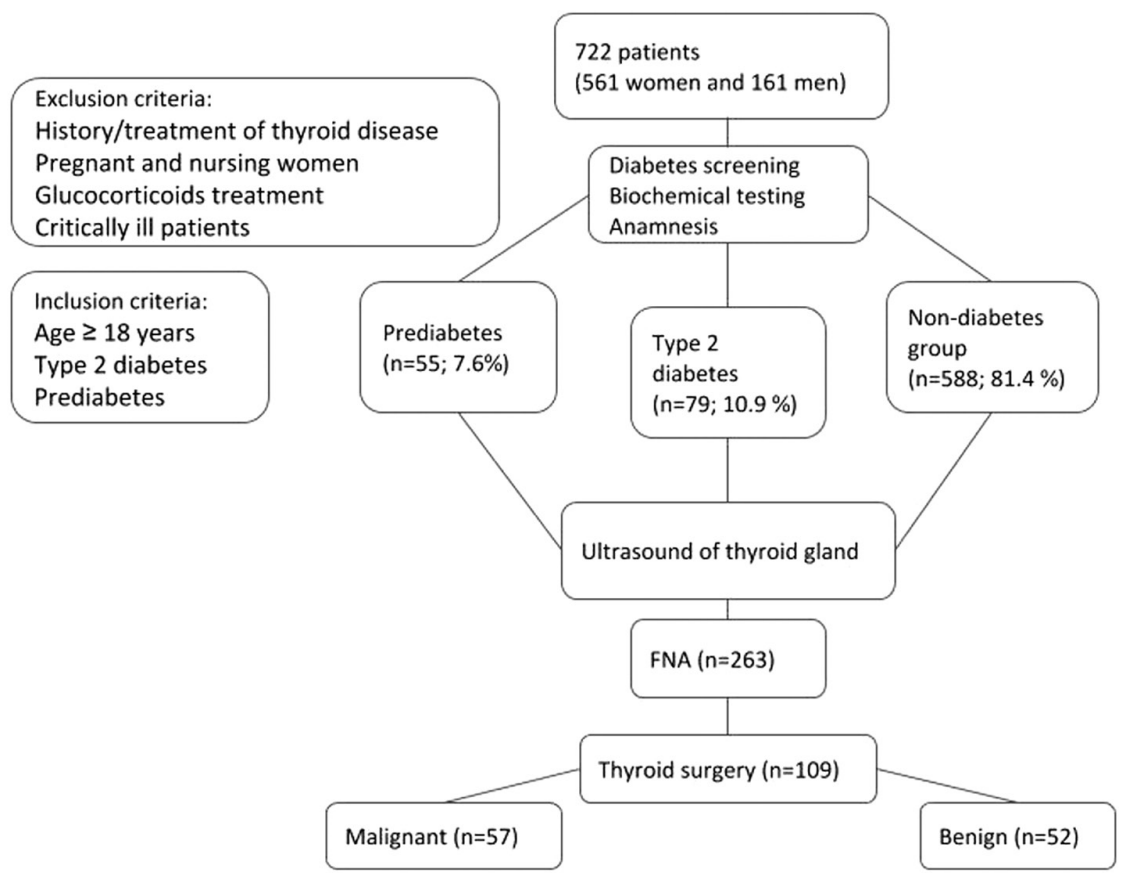

\section{Figure 1}

Flow chart of the study. or hyperthyroidism were re-checked for glucose disorders after reaching a euthyroid state. Basal blood samples for the determination of TSH, fT4, fT3, anti-thyroid peroxidase antibodies (anti-TPO), anti-thyroglobulin (anti-Tg), thyrotropin receptor antibodies (TRAbs), calcitonin, glucose, C-peptide, and hemoglobin A1c (HbA1c) were taken.

The patients were sorted to groups of prediabetes (PDM) ( $n=55 ; 7.5 \%)$, type 2 diabetes (T2DM) $(n=79$; $10.9 \%)$ or a non-diabetic group (NDM) $(n=588)$.

Fine-needle aspiration biopsy (FNA) was performed in 263 thyroid nodules using the pistol technique under US guidance. FNA was generally performed on thyroid nodules sized $>1 \mathrm{~cm}$. Thyroid nodules with suspicious US features such as hypoechogenicity, irregular or microlobulated margins, taller-than-wide, punctate echogenic foci and solid components were preferentially chosen for FNA. FNA was performed once for each thyroid nodule using a 20-gauge needle attached to a $20 \mathrm{~mL}$ syringe. Local anesthesia was not applied. In mixed nodules, solid areas were chosen. Aspirated material was expelled onto glass slides and sent for cytopathology examinations. MayGrünwald/Giemsa and hematoxylin and eosin stained specimens were evaluated by expert cytopathologists following the Bethesda System for Reporting Thyroid Cytopathology (TBSRTC) 2017: (1) nondiagnostic or unsatisfactory; (2) benign; (3) atypia of undetermined significance (AUS) or follicular lesion of undetermined significance (FLUS); (4) follicular neoplasm or suspicious for a follicular neoplasm; (5) suspicious for malignancy; and (6) malignant (19). US was performed at a frequency of $12.5 \mathrm{MHz}$ on a Phillips Epiq5.

By the end of the study, histological examinations had been done in 109 patients, with 57 findings being malignant (MS; 8.0\%) and 52 benign (BS).

Serum TSH (0.270-4.200 mUI/L), fT4 (12.00-22.00 $\mathrm{pmol} / \mathrm{L})$, fT3 (3.10-6.80 pmol/L), TRAbs (0.30-1.75 IU/L), calcitonin (1.0-4.8 ng/L) and C-peptide (268-1274 pmol/L) concentrations were measured using the ECLIA method (Roche). The HbA1C (20.0-42.0 $\mathrm{mmol} / \mathrm{mol})$ test was performed using an ion exchange HPLC method that is certified by the NGSP (www.ngsp.org) and standardized or traceable to the Diabetes Control and Complications Trial (DCCT) reference assay. Serum anti-Tg (0.01-120 IU/mL) and anti-TPO (0.01-40 IU/mL) were measured by ELISA (Aeskulisa). Glucose (3.9-5.6 mmol/L) was measured by a spectrophotometry (UV)-hexokinase method.

Statistical significance was set for $P$-values $<0.05$. Twoway ANOVA consisting of factors and diagnostic group (NDM, PDM, and T2DM) as well as age group (45-59 vs $>60$ years of age) with interactions between these factors was used to evaluate the effects of status and age. Due to the non-Gaussian data distribution and non-constant variance in most variables, the original continuous variables were transformed by power transformations prior to further processing to attain data symmetry and homoscedasticity (20). The homogeneity and distribution

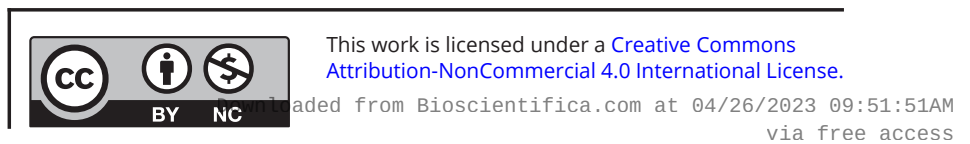


of transformed data were checked as described elsewhere $(21,22)$. Statistical software Statgraphics Centurion 18 from Statpoint (The Plains, VA, USA) was used for BoxCox transformations and ANOVA testing. Kruskal-Wallis multiple comparisons with Bonferroni correction for multiplicity were used for comparisons between diagnostic groups and age groups. The importance of individual predictors to discriminate between individual groups was evaluated using multivariate regression with a reduction of dimensionality known as orthogonal projections to latent structure (OPLS) for one predicted (dependent) variable in the model $(23,24)$. The method is effective in coping with the problem of multicollinearity within the matrix of predictors (high intercorrelations) where ordinary multiple regression fails to correctly evaluate such data (25).

\section{Results}

The patients $(n=722)$ were divided into groups of prediabetes (PDM) $(n=55 ; 7.5 \%$; age (years) medians with quartiles 67.4 (60.4-74.1), type 2 diabetes (T2DM) $(n=79$; $10.9 \%$; age 71.8 (63-76.6) and a non-diabetes group (NDM) $(n=588$; age 48.3 (36.6-64.5), with a significant difference for age between study groups $(P<0.001)$. The influence of age on the observed parameters and groups of PDM and T2DM in comparison to NDM was tested. BMI was significantly different between the study groups $(P<0.001)$ and followed by a significant impact of age on BMI $(P=0.004)$. Further, we observed a significant impact of age on TSH; especially, TSH level in NDM 2.02 (CI 95\% 1.79-2.26) vs PDM 4.17 (CI 95\% 2.5-6.91) $(P=0.028)$.

In general, the PDM/T2DM group of patients had higher values of glycemia, Hb1Ac and HOMA-IR $(P<0.001)$. The patients with T2DM had larger thyroid gland volume $(P=0.007)$ and higher fT4 $(P=0.011)$ in comparison to NDM. In contrast, the NDM group tended to have higher levels of anti-Tg $(P=0.064)$. The data are summarized in Table 1.

Thirty-three percent of patients with T2DM and especially PDM were newly diagnosed by our screening: $6.5 \%$ with T2DM and $72 \%$ with PDM, respectively. Thyroid nodule and/or multinodular thyroid gland (MNTG) were present in 66\% PDM, 62\% T2DM and 50\% NDM patients. Fine-needle aspiration (FNA) was carried out in 263 patients. FNA Bethesda findings were: (1) $(n=20$; $9.1 \%),(2)(n=170 ; 76.9 \%),(3)(n=12 ; 5.4 \%),(4) \quad(n=7$; $3.2 \%),(5)(n=6 ; 2.7 \%)$, and (6) $(n=6 ; 2.7 \%)$. Histological examination was done in 109 patients who underwent total thyroidectomy, with 57 findings malignant (8.0\%): 43 papillary thyroid carcinomas (75.4\%), 3 micromedullary thyroid carcinoma (MTC), 3 MTC, 2 anaplastic carcinoma, 1 follicular thyroid carcinoma, 1 oncocytic carcinoma, 1 metastasis and 3 poorly differentiated carcinoma. Malignant tumors according to diagnosis were $7 / 55$ in PDM (12.7\%), 9/79 in T2DM (11.4\%), and 41/588 in NDM (7.0\%). The percentage of malignant tumors did not significantly differ between the groups $\left(\chi^{2}\right.$ test $=0.461 ; P=0.794$ ).

Relevant positive predictors for T2DM $($ t-statistic $=25.87 ; P<0.01)$ and PDM $(21.69 ; P<0.01)$ contrary to $\mathrm{NDM}(-26.9 ; P<0.01)$ included thyroid volume $(\mathrm{mL})(4.79 ; P<0.01)$, multinodular thyroid gland (MNTG) $(4.83 ; P<0.01)$, thyroid nodule volume $(\mathrm{mL})$ (3.25; $P<0.01)$, BMI $(22.47 ; P<0.01)$, age $(16.98 ; P<0.01)$, smoking or history of smoking $(2.61 ; P<0.05)$ and nonthyroid cancer $(2.86 ; P<0.05)$, while negative relevant predictors included the occurrence of autoimmune thyroid disease (AITD) $(-2.01 ; P<0.05)$, anti-TPO $(-5.89$; $P<0.01)$, anti-Tg $(-5.75 ; P<0.01)$ and fT3 $(-2.86 ; P<0.05)$ (Table 2). Further, the relationships between the duration of PDM/T2DM and predictors were established. Due to the small number of patients in the PDM group, the PDM and T2DM groups were pooled for this analysis. Glycemia $(2.67 ; P<0.05)$ and $\mathrm{Hb} 1 \mathrm{Ac}(5.12 ; P<0.01)$ were positive relevant predictors for the duration of T2DM/PDM (4.52; $P<0.01)$. C-peptide $(-12.94 ; P<0.01)$, HOMA-IR $(-7.85 ; P<0.01)$, smoking or history of smoking $(-3.29$; $P<0.01)$ and AITD $(-2.3 ; P<0.05)$ were negative relevant predictors for the duration of T2DM and PDM (4.52; $P<0.01$ ) (Table 3).

Next, the group of patients undergoing thyroid surgery was analyzed, comparing malignant $(n=57$; MS) to benign thyroid histology $(n=52$; BS). Relevant positive predictors for MS with explained variability $35.5 \%$ after cross-validation (t-statistic $=5.45 ; P<0.01$ ) contrary to BS were TSH $(4.35 ; P<0.01)$, anti-Tg $(9.06 ; P<0.01)$ and FNA results $(4.94 ; P<0.01)$, while negative relevant predictors were thyroid gland volume $(-3.61 ; P<0.01)$ and thyroid nodule volume $(-3.55 ; P<0.01)$. Differences between group of patients with benign and malignant thyroid tumors are described in more detail in Table 4.

The history of non-thyroid cancer (NTC) was also analyzed in the PDM, T2DM and NDM groups. Thirty percent of T2DM and 10\% of PDM patients had a positive history for NTC in comparison to $8.4 \%$ of the NDM group excluding non-melanoma skin cancers. The most common types of cancers were colon, prostate, breast, 
Table 1 Characteristics of the study groups. Dependence of parameters on age and status (retransformed means with 95\% confidence limits).

\begin{tabular}{|c|c|c|c|c|}
\hline \multirow[b]{2}{*}{ Variable } & \multicolumn{3}{|c|}{ Group } & \multirow[b]{2}{*}{ ANOVA } \\
\hline & $\overline{N D M}$ & PDM & T2DM & \\
\hline $\mathrm{BMI}\left(\mathrm{kg} / \mathrm{m}^{2}\right)$ & $\begin{array}{l}26.2^{a, b} \\
(25.7,26.6)\end{array}$ & $\begin{array}{l}30.4^{a} \\
(28.4,32.7)\end{array}$ & $\begin{array}{c}31.7^{\mathrm{b}} \\
(30.4,33.2)\end{array}$ & $\begin{array}{l}\text { Age: } \mathrm{F}=3.5, P=0.062, \eta \mathrm{p} 2=0.0104 ; \text { Group: } \mathrm{F}=31.6 \\
P<0.001, \eta \mathrm{p} 2=0.159 ; \text { Age } \times \text { Group } \mathrm{F}=5.7, P=0.004 \\
\eta p 2=0.0327\end{array}$ \\
\hline $\begin{array}{l}\text { Thyroid gland } \\
\text { volume }(\mathrm{mL})\end{array}$ & $\begin{array}{l}11.7^{a} \\
(11.1,12.3)\end{array}$ & $\begin{array}{l}12.4 \\
(10.2,15.5)\end{array}$ & $\begin{array}{c}15^{\mathrm{a}} \\
(13.2,17.2)\end{array}$ & $\begin{array}{l}\text { Age: } \mathrm{F}=0.5, P=0.477, \eta \mathrm{p} 2=0.00123 ; \text { Group: } \mathrm{F}=5.1 \\
P=0.007, \eta \mathrm{p} 2=0.024 ; \text { Age } \times \text { Group } \mathrm{F}=0.1, P=0.928 \\
\eta \mathrm{p} 2=0.000364\end{array}$ \\
\hline $\begin{array}{l}\text { Thyroid nodule } \\
\text { volume }(\mathrm{mL})\end{array}$ & $\begin{array}{l}0.5 \\
(0.4,0.7)\end{array}$ & $\begin{array}{l}0.5 \\
(0.1,1.5)\end{array}$ & $\begin{array}{c}1.0 \\
(0.51,2.0)\end{array}$ & $\begin{array}{l}\text { Age: } F=1.3, P=0.262, \eta p 2=0.00301 ; \text { Group: } F=1.1 \\
P=0.345, \eta p 2=0.00507 ; \text { Age } \times \text { Group } F=1, P=0.37 \\
\eta p 2=0.00475\end{array}$ \\
\hline $\begin{array}{l}\text { TSH } \\
(\mathrm{mlU} / \mathrm{L})\end{array}$ & $\begin{array}{l}2.02^{\mathrm{a}} \\
(1.79,2.26)\end{array}$ & $\begin{array}{l}4.17^{\mathrm{a}} \\
(2.5,6.91)\end{array}$ & $\begin{array}{c}2.06 \\
(1.57,2.68)\end{array}$ & $\begin{array}{l}\text { Age: } \mathrm{F}=8.5, P=0.004, \eta \mathrm{p} 2=0.0206 ; \text { Group: } \mathrm{F}=2.7 \\
P=0.066, \eta \mathrm{p} 2=0.0134 ; \text { Age } \times \text { Group } \mathrm{F}=3.6, P=0.028 \\
\eta \mathrm{p} 2=0.0176\end{array}$ \\
\hline $\begin{array}{l}\text { fT4 } \\
\text { (pmol/l) }\end{array}$ & $\begin{array}{l}14.5^{\mathrm{a}} \\
(14.2,14.7)\end{array}$ & $\begin{array}{l}14.6 \\
(13.4,15.9)\end{array}$ & $\begin{array}{c}15.6^{\mathrm{a}} \\
(15,16.3)\end{array}$ & $\begin{array}{l}\text { Age: } \mathrm{F}=0.5, P=0.489, \eta \mathrm{p} 2=0.00134 ; \text { Group: } \mathrm{F}=4.5 \\
P=0.011, \eta \mathrm{p} 2=0.0247 ; \text { Age } \times \text { Group } \mathrm{F}=0.1, P=0.874 \\
\eta p 2=0.000748\end{array}$ \\
\hline $\begin{array}{l}\text { fT3 } \\
(\mathrm{pmol} / \mathrm{l})\end{array}$ & $\begin{array}{l}4.66 \\
(4.58,4.73)\end{array}$ & $\begin{array}{l}4.74 \\
(4.44,5.05)\end{array}$ & $\begin{array}{c}4.82 \\
(4.62,5.02)\end{array}$ & $\begin{array}{l}\text { Age: } \mathrm{F}=4.1, P=0.045, \eta \mathrm{p} 2=0.0167 ; \text { Group: } \mathrm{F}=0.9 \\
P=0.406, \eta \mathrm{p} 2=0.00753 ; \text { Age } \times \text { Group } \mathrm{F}=0.8, P=0.474 \\
\eta \mathrm{p} 2=0.00622\end{array}$ \\
\hline anti-TPO (IU/mL) & $\begin{array}{l}0.92 \\
(0.77,1.09)\end{array}$ & $\begin{array}{l}0.68 \\
(0.20,1.39)\end{array}$ & $\begin{array}{c}0.48 \\
(0.22,0.78)\end{array}$ & $\begin{array}{l}\text { Age: } \mathrm{F}=0.3, P=0.617, \eta \mathrm{p} 2=0.0006 ; \text { Group: } \mathrm{F}=2.4 \\
P=0.09, \eta \mathrm{p} 2=0.0115 ; \text { Age } \times \text { Group } \mathrm{F}=0.5, P=0.601 \\
\eta p 2=0.00243\end{array}$ \\
\hline anti-Tg (IU/mL) & $\begin{array}{l}0.40^{\mathrm{a}} \\
(0.34,0.48)\end{array}$ & $\begin{array}{l}0.27 \\
(0.05,0.56)\end{array}$ & $\begin{array}{c}0.19^{\mathrm{a}} \\
(0.08,0.33)\end{array}$ & $\begin{array}{l}\text { Age: } \mathrm{F}=0.8, P=0.371, \eta \mathrm{p} 2=0.00191 ; \text { Group: } \mathrm{F}=2.8 \\
P=0.064, \eta \mathrm{p} 2=0.013 ; \text { Age } \times \text { Group: } \mathrm{F}=0.3, P=0.738 \\
\eta \mathrm{p} 2=0.00145\end{array}$ \\
\hline $\begin{array}{l}\text { TRAbs } \\
\text { (IU/L) }\end{array}$ & $\begin{array}{l}0.06 \\
(0.05,0.07)\end{array}$ & $\begin{array}{l}0.08 \\
(0.03,0.16)\end{array}$ & $\begin{array}{c}0.06 \\
(0.04,0.09)\end{array}$ & $\begin{array}{l}\text { Age: } \mathrm{F}=1.1, P=0.3, \eta \mathrm{p} 2=0.00257 ; \text { Group: } \mathrm{F}=0.2, \\
P=0.844, \eta \mathrm{p} 2=0.000814 ; \text { Age } \times \text { Group } \mathrm{F}=0.8, P=0.461 \\
\eta \mathrm{p} 2=0.0037\end{array}$ \\
\hline C peptide (pmol/L) & $\begin{array}{l}713 \\
(646,789)\end{array}$ & $\begin{array}{l}817 \\
(660,1020)\end{array}$ & $\begin{array}{c}886 \\
(769,1020)\end{array}$ & $\begin{array}{l}\text { Age: } \mathrm{F}=0.4, \mathrm{P}=0.52, \eta \mathrm{p} 2=0.00307 ; \text { Group: } \mathrm{F}=2.4 \\
P=0.095, \eta \mathrm{p} 2=0.0343 ; \text { Age } \times \text { Group } \mathrm{F}=0.4, P=0.667 \\
\eta p 2=0.00599\end{array}$ \\
\hline $\begin{array}{l}\text { Glycaemia (3.9-5.6) } \\
\text { (mmol/L) }\end{array}$ & $\begin{array}{l}5.06 \\
(5.02,5.11)\end{array}$ & $\begin{array}{l}6.09 \\
(5.83,6.39)\end{array}$ & $\begin{array}{c}7.6 \\
(7.23,8.06)\end{array}$ & $\begin{array}{l}\text { Age: } F=0, P=0.868, \eta p 2=0.0000965 ; \text { Group: } F=236.7 \\
P<0.001, \eta p 2=0.621 ; \text { Age } \times \text { Group } F=0.7, P=0.508 \\
\eta p 2=0.00468, P>C, D>P, D>C\end{array}$ \\
\hline $\begin{array}{l}\mathrm{HbA} 1 \mathrm{c}(20.0-42.0) \\
(\mathrm{mmol} / \mathrm{mol})\end{array}$ & $\begin{array}{l}36.1^{a, b} \\
(35.5,36.6)\end{array}$ & $\begin{array}{l}38.9^{b, c} \\
(37.4,40.7)\end{array}$ & $\begin{array}{c}50.7^{\mathrm{a}, \mathrm{c}}(48.2 \\
53.8)\end{array}$ & $\begin{array}{l}\text { Age: } \mathrm{F}=3.2, P=0.074, \eta \mathrm{p} 2=0.0166 ; \text { Group: } \mathrm{F}=98.8 \\
P<0.001, \eta \mathrm{p} 2=0.509 ; \text { Age } \times \text { Group } \mathrm{F}=1.8, P=0.175 \\
\eta p 2=0.0181\end{array}$ \\
\hline HOMA-IR & $\begin{array}{l}1.58^{\mathrm{a}} \\
(1.43,1.73)\end{array}$ & $\begin{array}{l}1.9 \\
(1.54,2.36)\end{array}$ & $\begin{array}{c}2.37^{a} \\
(2.05,2.76)\end{array}$ & $\begin{array}{l}\text { Age: } \mathrm{F}=1.6, P=0.212, \eta \mathrm{p} 2=0.0116 ; \text { Group: } \mathrm{F}=8.3 \\
P<0.001, \eta \mathrm{p} 2=0.11 ; \text { Age } \times \text { Group } \mathrm{F}=0.3, P=0.724 \\
\eta \mathrm{p} 2=0.00481\end{array}$ \\
\hline $\begin{array}{l}\text { HOMAB } \\
(\%)\end{array}$ & $\begin{array}{l}126^{a, b} \\
(118,133)\end{array}$ & $\begin{array}{l}101^{\mathrm{a}, \mathrm{c}} \\
(85.8,116)\end{array}$ & $\begin{array}{c}61^{\mathrm{b}, \mathrm{c}} \\
(51.8,70.4)\end{array}$ & $\begin{array}{l}\text { Age: } \mathrm{F}=0.2, P=0.658, \eta \mathrm{p} 2=0.00152 ; \text { Group: } \mathrm{F}=41.2 \\
P<0.001, \eta \mathrm{p} 2=0.388 ; \text { Age } \times \text { Group } \mathrm{F}=2.2, P=0.117 \\
\eta \mathrm{p} 2=0.0325\end{array}$ \\
\hline $\begin{array}{l}\text { HOMAS } \\
(\%)\end{array}$ & $\begin{array}{l}63.8^{\mathrm{a}} \\
(57.8,70.2)\end{array}$ & $\begin{array}{l}52.8 \\
(42.2,65.2)\end{array}$ & $\begin{array}{c}42^{\mathrm{a}} \\
(35.9,48.7)\end{array}$ & $\begin{array}{l}\text { Age: } \mathrm{F}=1.6, P=0.212, \eta \mathrm{p} 2=0.0116 ; \text { Group: } \mathrm{F}=8.3 \\
P<0.001, \eta \mathrm{p} 2=0.11 ; \text { Age } \times \text { Group } \mathrm{F}=0.3, P=0.722 \\
\eta p 2=0.00485\end{array}$ \\
\hline
\end{tabular}

anti-Tg, anti-thyroglobulin antibodies; anti-TPO, anti-thyroid peroxidase antibodies; HOMA-B (\%), steady state $\beta$ cell function; HOMA-IR, the homeostatic model assessment of insulin resistance; HOMA-S (\%), insulin sensitivity; NDM, non-diabetes group; PDM, prediabetes; T2DM, type 2 diabetes; TRAbs, TSH receptor autoantibodies.

Significance for multiple comparisons with Bonferroni correction ( $P<0.05)$. Glycaemia (3.9-5.6) (mmol/L); 5.06a,b (5.02, 5.11); 6.09 (7.23, 8.06); Age: $\mathrm{F}=0, P=0.868, \eta p 2=0.0000965 ;$ Group: $\mathrm{F}=236.7, P<0.001, \eta \mathrm{p} 2=0.621$; Age $\times$ Group $\mathrm{F}=0.7, P=0.508, \eta p 2=0.00468$.

melanoma and urothelial carcinoma. Four patients in the PDM/T2DM group had cancer duplicity. Relevant positive predictors for NTC with explained variability 9\% after cross-validation (t-statistic 3.01; $P<0.01$ ) were T2DM (24.99; $P<0.01)$, duration of PDM/T2DM (13.30; $P<0.01)$, male gender $(6.51 ; P<0.01)$, age $(12.95 ; P<0.01)$,
BMI (13.68; $P<0.01)$, smoking or history of smoking (6.01; $P<0.01)$, multinodular thyroid gland (12.00; $P<0.01)$, thyroid nodule volume $(6.78 ; P<0.01)$ and glycemia $(21.13 ; P<0.01)$. In contrast, relevant negative predictors for NTC were anti-TPO $(-3.90 ; P<0.01)$ and anti-Tg $(-3.84 ; P<0.01)$. 
Table 2 Relationships between groups of patients with prediabetes (PDM), type 2 diabetes (T2DM), and a control group (nondiabetes group; NDM) and predictors for the first predictive component as evaluated by the O2PLS model (for details see Statistical analysis).

\begin{tabular}{|c|c|c|c|c|c|}
\hline \multirow{4}{*}{$\begin{array}{l}\text { Relevant predictors } \\
\text { (matrix X) }\end{array}$} & \multirow{3}{*}{$\frac{\text { Variable }}{\text { Male }}$} & \multicolumn{4}{|c|}{ Predictive component } \\
\hline & & \multirow{2}{*}{ Component loading } & \multirow{2}{*}{$\begin{array}{c}t \text {-statistics } \\
4.53\end{array}$} & \multicolumn{2}{|c|}{$R^{\mathrm{a}}$} \\
\hline & & & & 0.280 & c \\
\hline & Age & 0.460 & 16.98 & 0.708 & c \\
\hline & $\mathrm{BMI}$ & 0.388 & 22.47 & 0.604 & c \\
\hline & Smoking & 0.079 & 2.61 & 0.112 & b \\
\hline & NTC & 0.198 & 2.86 & 0.295 & b \\
\hline & MNTG & 0.176 & 4.83 & 0.249 & c \\
\hline & Thyroid nodule volume & 0.090 & 3.25 & 0.116 & c \\
\hline & Thyroid gland volume & 0.135 & 4.79 & 0.201 & c \\
\hline & AITD & -0.056 & -2.01 & -0.076 & $\mathrm{~b}$ \\
\hline & fT3 & -0.071 & -2.86 & -0.114 & b \\
\hline & anti-TPO & -0.144 & -5.89 & -0.201 & c \\
\hline & anti-Tg & -0.132 & -5.75 & -0.180 & c \\
\hline & Glycaemia & 0.655 & 52.63 & 0.885 & c \\
\hline \multirow[t]{3}{*}{ (matrix Y) } & T2DM & 0.597 & 25.87 & 0.508 & c \\
\hline & PDM & 0.355 & 21.69 & 0.255 & c \\
\hline & NDM & -0.721 & -26.90 & -0.583 & c \\
\hline Explained variability & & \multicolumn{4}{|c|}{$22 \%$ (20.8\% after cross-validation) } \\
\hline
\end{tabular}

AITD, autoimmune thyroid disease; anti-Tg, anti-thyroglobulin antibodies; anti-TPO, anti-thyroid peroxidase antibodies; MNTG, multinodular thyroid gland; NTC, non-thyroid cancer; Smoking or history of smoking.

${ }^{a} R$, Component loadings expressed as correlation coefficients with predictive component, ${ }^{b} P<0.05,{ }^{c} P<0.01$.

\section{Discussion}

Generally, there is a higher prevalence of thyroid disorders among diabetic patients compared with the general population (10.8\% vs $6.6 \%$ ) (26). Epidemiological studies suggest that the incidence of thyroid cancer (TC) is dependent on modifiable risk factors such as environmental carcinogens, dietary habits, and lifestyle $(27,28)$. In our study, we focused on the links between prediabetes, type 2 diabetes and thyroid disease. Specifically, we concentrated on the risk of TC and the modifiable factors, diabetes, obesity and insulin resistance.

In our study, the typical patient with PDM or DM was an elderly, overweight or obese man with benign multinodular goiter. In addition, smoking or ex-smoking was a common history of these patients. On the other hand, the typical control proband was younger with a higher incidence of autoimmune thyroid disorder. Our findings are supported by other studies showing that type 2 diabetes is not convincingly associated with the prevalence of hypo- or hyperthyroidism or the presence

Table 3 Relationships between the duration of prediabetes (PDM) and type 2 diabetes (T2DM) and predictors for the first predictive component as evaluated by the O2PLS model (for details see the Statistical analysis section).

\begin{tabular}{|c|c|c|c|c|c|}
\hline & & & e componen & & \\
\hline & Variable & Component loading & $t$-statistics & $R^{a}$ & \\
\hline Relevant predictors & AITD & -0.189 & -2.30 & -0.334 & b \\
\hline$($ matrix $X)$ & Smoking & -0.140 & -3.29 & -0.249 & c \\
\hline & C-peptide & -0.497 & -12.94 & -0.889 & c \\
\hline & Glycemia & 0.223 & 2.67 & 0.391 & b \\
\hline & $\mathrm{HbA} 1 \mathrm{c}$ & 0.254 & 5.12 & 0.427 & c \\
\hline & HOMA-IR & -0.442 & -7.85 & -0.793 & c \\
\hline & HOMA-B & -0.479 & -8.85 & -0.855 & c \\
\hline & HOMA-S & 0.443 & 7.72 & 0.794 & c \\
\hline (matrix Y) & Duration of PDM/T2DM & 1.000 & 4.52 & 0.529 & c \\
\hline Explained variability & & 27.9 & ter cross- $v$ & & \\
\hline
\end{tabular}

AITD, autoimmune thyroid disease; HOMA-B, steady state beta cell function; HOMA-IR, the homeostatic model assessment of insulin resistance; HOMA-S, insulin sensitivity; Smoking or history of smoking.

${ }^{a} R$, Component loadings expressed as correlation coefficients with predictive component, ${ }^{b} P<0.05, c P<0.01$.

https://ec.bioscientifica.com

https://doi.org/10.1530/EC-20-0180
(C) 2020 The authors Published by Bioscientifica Ltd

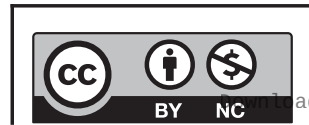

This work is licensed under a Creative Commons Attribution-NonCommercial 4.0 International License. ded from Bioscientifica.com at 04/26/2023 09:51:51AM via free access 
Table 4 Differences between group of patients with benign (BS) and malignant thyroid tumors (MS) for metric variables (Mann-Whitney U-test).

\begin{tabular}{|c|c|c|}
\hline \multirow[b]{2}{*}{ Variable } & \multicolumn{2}{|c|}{ BS } \\
\hline & Median (quartiles) & Mean (s.D.) \\
\hline Age & $47(38.8,65.3)$ & 49.8 (15.9) \\
\hline $\mathrm{BMI}$ & $25.2(22.2,29.9)$ & $27(6.61)$ \\
\hline TN & $8.2(2,16.5)$ & $12.8(16.8)$ \\
\hline TG & $24.5(16.6,34.8)$ & $32.8(37.4)$ \\
\hline $\mathrm{TSH}$ & $1.08(0.117,1.96)$ & $1.23(1.09)$ \\
\hline fT4 & $15.7(14.1,18.4)$ & $21.8(16.4)$ \\
\hline fT3 & $5(4.76,5.79)$ & 7.99 (8.53) \\
\hline anti-TPO & $5.02(1.88,12)$ & $31(68.6)$ \\
\hline anti-Tg & $0.86(0.36,3.55)$ & $4.74(9.61)$ \\
\hline TRAbs & $0.39(0.3,10.8)$ & $5.77(9.88)$ \\
\hline C-peptide & $818(598,1110)$ & 895 (516) \\
\hline Glycaemia & $5.31(4.87,5.58)$ & $5.62(1.55)$ \\
\hline $\mathrm{Hb} 1 \mathrm{Ac}$ & $41(36,53)$ & $48.9(22.8)$ \\
\hline HOMA-IR & $2.26(1.61,2.52)$ & $2.39(1.28)$ \\
\hline Calcitonin & $2(0.9,3.35)$ & $2.84(2.67)$ \\
\hline
\end{tabular}

\begin{tabular}{c}
\hline \\
\hline MS \\
\hline Median (quartiles) \\
\hline $53(39,67)$ \\
$26.8(22.5,30.1)$ \\
$1.8(0.22,7.3)$ \\
$17(10.8,27.4)$ \\
$2.14(1.25,3.37)$ \\
$15.4(14,16.4)$ \\
$4.8(4.5,5.21)$ \\
$6.1(2.98,144)$ \\
$12.8(1.63,33.8)$ \\
$0.32(0.3,0.6)$ \\
$729(434,887)$ \\
$5.2(4.9,5.58)$ \\
$36(34.4,38.2)$ \\
$1.61(0.98,2.35)$ \\
$3.2(1.3,6.93)$
\end{tabular}

\begin{tabular}{c} 
Mean (S.D.) \\
\hline $53(16.8)$ \\
$26.7(5.27)$ \\
$6.24(14.4)$ \\
$22.5(17.5)$ \\
$3.86(7.75)$ \\
$15(3.31)$ \\
$4.83(0.624)$ \\
$154(317)$ \\
$284(1330)$ \\
$0.915(1.82)$ \\
$866(669)$ \\
$5.44(1.1)$ \\
$37.2(7.14)$ \\
$2(1.58)$ \\
$20(69.8)$ \\
\hline
\end{tabular}

\begin{tabular}{r}
$P$-value \\
\hline 0.402 \\
0.786 \\
$<0.001$ \\
0.021 \\
$<0.001$ \\
0.145 \\
0.071 \\
0.259 \\
0.001 \\
0.293 \\
0.484 \\
0.792 \\
0.080 \\
0.178 \\
0.184
\end{tabular}

anti-Tg, anti-thyroglobulin antibodies; anti-TPO, anti-thyroid peroxidase antibodies; HOMA-IR, the homeostatic model assessment of insulin resistance; TG, thyroid gland volume $(\mathrm{mL}) ; \mathrm{TN}$, thyroid nodule volume $(\mathrm{mL}) ;$ TRAbs, TSH receptor autoantibodies.

of anti-TPO positivity $(29,30,31)$. After statistical analyses of PDM/T2DM duration on relevant predictors, we found that the typical course of PDM/T2DM is a gradual decline of insulin secretion, progression of hyperglycemia and worsening of glucose control as measured by Hb1Ac, with insulin sensitivity assessed by HOMA-S at a maximum. Finally, autoimmune thyroid disorder did not develop frequently during the course of prediabetes and type 2 diabetes.

In a multivariate analysis, insulin resistance, and not $\mathrm{TSH}$, determines the thyroid nodules development and higher thyroid volume in comparison to non-insulin resistant controls $(32,33,34)$. Our study brings similar findings, with the group of prediabetic and diabetic patients having both larger thyroid gland volume and larger thyroid nodule size. We have to emphasize that ageing is crucial in this pathogenesis. The key role of TSH signaling in thyroid carcinogenesis has been supported by large epidemiological studies showing a strong association between serum TSH levels and TC development and progression. On the other hand, it is known that TSH levels can be increased in differentiated thyroid carcinoma (DTC) compared to benign thyroid disease (BTD), but not in comparison to the background population. A lower TSH among patients with BTD may be due to co-existing functional nodular autonomy $(35,36,37)$. This bias can lead to misinterpretations of a positive relation between higher TSH and risk of DTC. In our study, we did not observe any difference in TSH levels between the PDM, T2DM and NDM groups of patients. Our results are supported by a large population-based study that showed no convincing evidence that people with type 2 diabetes are at increased risk of hypothyroidism (29). However, we observed significantly higher levels of TSH in patients undergoing thyroid surgery with histologically proven malignancy in comparison to lower TSH levels in the benign group. Multinodular goiter, multinodular toxic goiter, toxic nodule and Graves-Basedow thyrotoxicosis were the reasons for our benign surgery results. Generally, toxic nodules have a very low risk of malignancy (6). This clearly explains the TSH level differences between the MS and BS groups, and TSH levels have not generally been helpful in the prediction of TC risk.

Additionally, we observed lower fT3 levels but higher fT4 levels in the normal range in PDM and T2DM patients. There are some studies supporting our findings. One explanation can be genetic polymorphism in the selenodeiodinases D2 in diabetic patients. The major role of D2 is to control the intracellular T3 concentration, its accessibility to the nucleus, and the saturation of the nuclear T3 receptor in target tissues. In some diabetic patients lower D2 activity is present, and therefore, intrinsic thyroid disease is expected with possible metabolic consequences $(38,39)$.

Case-control and cohort studies have confirmed an increased TC risk in approximately $20 \%$ in diabetic patients, independently of geographic region, study design, and quality analysis. Despite the high heterogeneity among studies, the observation that the risk is increased among diabetic women, but not among men, has been always https://ec.bioscientifica.com

https://doi.org/10.1530/EC-20-0180 (c) 2020 The authors Published by Bioscientifica Ltd
This work is licensed under a Creative Commons Attribution-NonCommercial 4.0 International License. ed from Bioscientifica.com at 04/26/2023 09:51:51AM via free access 
confirmed. In addition, the TC risk associated with DM is more evident in geographic areas of the world with high rates of TC $(40,41,42)$.

In our study, we did not observe any TC risk difference between PDM and T2DM in comparison to NDM similarly to other study published previously (43). We suppose that one of the explanations is the overdiagnosis of TC in diabetics. Diabetic patients are more prone to be screened for thyroid dysfunction, thus contributing to the increased detection of thyroid cancer in this population. Further, they have larger thyroid gland volume and larger thyroid nodule size, perhaps leading to earlier TC manifestation and detection. Generally, thyroid incidentalomas are very frequent findings (26). In our study, we used histologically proven results as the gold reference standard to compare patients with TC and control group. We believe that sample selection bias of the control group could be the reason for different study results in comparison to previous studies. Finally, in our study the patients with proven TC had a smaller thyroid volume and thyroid nodule volume, in contrast to diabetic patients with typical multinodular goiter.

As mentioned previously, we did not observe a higher incidence of TC in PDM/T2DM patients compared to NDM. In contrast, $30 \%$ of T2DM and $10 \%$ of PDM patients had a positive history for non-thyroid cancer (NTC) in comparison to $8.4 \%$ in NDM. Our study also supports the relation between diabetes and cancer. The incidence of NTC increases with the duration of PDM/ T2DM. As previously described, the positivity of antiTPO and/or anti-Tg were relevant negative predictors for both PDM/T2DM and NTC. Perhaps, patients with autoimmune thyroid disorder have a different background in comparison to diabetics and the NTC group of patients. Furthermore, multinodular thyroid gland and larger thyroid nodules were more often present in the NTC group. This may support the idea of the growth stimulation of various types of both benign and malignant tumors in diabetics.

Epidemiological studies, followed by the systematic review suggest that obesity is associated with an increased risk of thyroid cancer; however, the relationships between obesity and thyroid cancer stage or behavior are uncertain $(44,45)$. In our study, we did not observe higher BMI $(P=0.786)$ or insulin resistance $(P=0.178)$ in group of patients with thyroid carcinoma. In contrast, BMI was one of relevant positive predictors for non-thyroid cancers. We suppose that obesity is not significantly related to thyroid cancer compared to other malignancies.
Finally, we would like to emphasize that TC differs from other tumors in age distribution, with the most commonly affected group of patients in the economic productive age of 24-60 years, while in other malignancies the risk of carcinoma increases continuously with age. In contrast, in developed countries, more than half of the people with type 2 diabetes mellitus are older than 65 years and only $8 \%$ are less than 44 years of age (46).

One limitation of our study is the difference between the study groups in age - the PDM and T2DM group of patients were older in comparison to the NDM group. For that reason, we adjusted the data on age, but we did not observe significant interactions between age and the tested parameters except BMI and TSH. Another limitation is the small size of the prediabetic group. This may have been the reason why some significant results seen in T2DM were not significant for PDM. The number of patients with histologically proven thyroid tumors was limited; however, the sample size reached sufficient statistical power. Further, C-peptide levels were not available in all patients, but it was sufficient for the statistical analysis. However, a strength of our study is the prospective design with active screening for glucose disorders and the use of histology as the reference standard. It should be noted that in some previous studies the diagnosis of prediabetes/diabetes was ascertained by a self-administered questionnaire. In our study $72 \%$ patients with PDM and 6.5\% with T2DM were diagnosed by our active screening. Self-reported PDM and T2DM can be quite misleading, with some studies supporting our findings of undiagnosed glucose disorders of up to $45.8 \%$ globally (47). Our study also includes prediabetes, and to our knowledge, limited data is available for this subgroup of patients. Taken together, we believe that our study can, to some extent, explain the inconsistencies in previously published studies focused on diabetes and links to thyroid cancer and other thyroid diseases.

\section{Conclusions}

In the most insulin-resistant subjects, the diabetic and prediabetic groups, we did not observe an increased risk for thyroid cancer, in spite of the well-established increased risk for other malignancies. Structural and benign changes such as larger and multinodular thyroid gland, in comparison to autoimmune thyroid disease, were present more often in patients with prediabetes and 2 type diabetes. Intrinsic thyroid disease cannot be ruled out even under euthyroidism in these patients.

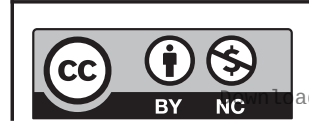

This work is licensed under a Creative Commons Attribution-NonCommercial 4.0 International License. ded from Bioscientifica.com at 04/26/2023 09:51:51AM 
Reasons for this finding may simply be overdiagnosis and/or stimulation of tumor growth with earlier manifestations in pre/diabetics or other unknown reasons remaining to be clarified.

\section{Declaration of interest}

The authors declare that there is no conflict of interest that could be perceived as prejudicing the impartiality of the research reported

\section{Funding}

This work was supported by Ministry of Health, Czech Republic - conceptual development of research organization (Institute of Endocrinology - EU, 00023761).

\section{Author contribution statement}

Tereza Grimmichova conceived of the presented idea. Martin Haluzik and Karel Vondra supervised the findings of this work. Martin Hill and Petr Matucha analyzed the data. All authors discussed the results and contributed to the final manuscript.

\section{Acknowledgements}

The authors are grateful to the director of Institute of Endocrinology and Ass. Prof RNDr. Bela Bendlova, Csc., Mgr. Josef Vcelak and MUDr. Katerina Dvorakova for general support and manuscript preparation.

\section{References}

1 Ito Y, Nikiforov YE, Schlumberger M \& Vigneri R. Increasing incidence of thyroid cancer: controversies explored. Nature Reviews. Endocrinology 20139 178-184. (https://doi.org/10.1038/ nrendo.2012.257)

2 Roti E, Degli Uberti EC, Bondanelli \& Braverman LE. Thyroid papillary microcarcinoma: a descriptive and meta-analysis study. European Journal of Endocrinology 2008159 659-673. (https://doi org/10.1530/EJE-07-0896)

3 Ferlay J, Shin HR, Bray F, Forman D, Mathers C \& Parkin DM. Estimates of worldwide burden of cancer in 2008: GLOBOCAN 2008. International Journal of Cancer 2010127 2893-2917. (https://doi. org/10.1002/ijc.25516)

4 La Vecchia C \& Negri E. Thyroid cancer: the thyroid cancer epidemic - overdiagnosis or a real increase? Nature Reviews Endocrinology 2017 13 318-319. (https://doi.org/10.1038/nrendo.2017.53)

5 Enewold L, Zhu K, Ron E, Marrogi AJ, Stojadinovic A, Peoples GE $\&$ Devesa SS. Rising thyroid cancer incidence in the United States by demographic and tumor characteristics, 1980-2005. Cancer Epidemiology, Biomarkers \& Prevention 200918 784-791. (https://doi. org/10.1158/1055-9965.EPI-08-0960)

6 Haugen BR, Alexander EK, Bible KC, Doherty GM, Mandel SJ, Nikiforov YE, Pacini F, Randolph GW, Sawka AM, Schlumberger M, et al. 2015 American Thyroid Association management guidelines for adult patients with thyroid nodules and differentiated thyroid cancer: the American Thyroid Association guidelines task force on thyroid nodules and differentiated thyroid cancer. Thyroid 201626 1-133. (https://doi.org/10.1089/ thy.2015.0020)

7 Bergman A, Heindel JJ, Kasten T, Kidd KA, Jobling S, Neira M, Zoeller RT, Becher G, Bjerregaard P, Bornman R, et al. The impact of endocrine disruption: a consensus statement on the state of the science. Environmental Health Perspectives 2013121 A104-A106. (https://doi.org/10.1289/ehp.1205448)

8 Harach HR, Franssila KO \& Wasenius VM. Occult papillary carcinoma of the thyroid. A normal finding in Finland. A systematic autopsy study. Cancer 198556 531-538. (https:// doi.org/10.1002/1097-0142(19850801)56:3<531::aidcncr2820560321>3.0.co;2-3)

9 Anil C, Goksel S \& Gursoy A. Hashimoto's thyroiditis is not associated with increased risk of thyroid cancer in patients with thyroid nodules: a single-center prospective study. Thyroid 201020 601-606. (https://doi.org/10.1089/thy.2009.0450)

10 Singh B, Shaha AR, Trivedi H, Carew JF, Poluri A \& Shah JP. Coexistent Hashimoto's thyroiditis with papillary thyroid carcinoma: impact on presentation, management, and outcome. Surgery 1999126 1070-1076; discussion 1076. (https://doi.org/10.1067/ msy.2099.101431)

11 Bhargav PR, Mishra A, Agarwal G, Agarwal A, Pradhan PK, Gambhir S, Verma AK \& Mishra SK. Long-term outcome of differentiated thyroid carcinoma: experience in a developing country. World Journal of Surgery 201034 40-47. (https://doi. org/10.1007/s00268-009-0293-y)

12 Lupoli G, Vitale G, Caraglia M, Fittipaldi MR, Abbruzzese A, Tagliaferri P \& Bianco AR. Familial papillary thyroid microcarcinoma: a new clinical entity. Lancet 1999353 637-639. (https://doi. org/10.1016/S0140-6736(98)08004-0)

13 La Vecchia C, Negri E, Franceschi S, D’Avanzo B \& Boyle P. A casecontrol study of diabetes mellitus and cancer risk. British Journal of Cancer 199470 950-953. (https://doi.org/10.1038/bjc.1994.427)

14 Paes JE, Hua K, Nagy R, Kloos RT, Jarjoura D \& Ringel MD. The relationship between body mass index and thyroid cancer pathology features and outcomes: a clinicopathological cohort study. Journal of Clinical Endocrinology \& Metabolism 201095 4244-4250. (https://doi. org/10.1210/jc.2010-0440)

15 Zamrazil V, Bilek R, Cerovska J \& Delange F. The elimination of iodine deficiency in the Czech Republic: the steps toward success. Thyroid 200414 49-56. (https://doi. org/10.1089/105072504322783849)

16 American Diabetes Association. 2. Classification and diagnosis of diabetes: standards of medical care in diabetes - 2018. Diabetes Care 201841 S13-S27. (https://doi.org/10.2337/dc18-S002)

17 Inzucchi SE, Bergenstal RM, Buse JB, Diamant M, Ferrannini E, Nauck M, Peters AL, Tsapas A, Wender R \& Matthews DR. Management of hyperglycaemia in type 2 diabetes, 2015: a patientcentred approach. Update to a position statement of the American Diabetes Association and the European Association for the Study of Diabetes. Diabetologia 201558 429-442. (https://doi.org/10.1007/ s00125-014-3460-0)

18 Ohkura T, Shiochi H, Fujioka Y, Sumi K, Yamamoto N, Matsuzawa K, Izawa S, Kinoshita H, Ohkura H, Kato M, et al. 20/(fasting C-peptide $\times$ fasting plasma glucose) is a simple and effective index of insulin resistance in patients with type 2 diabetes mellitus: a preliminary report. Cardiovascular Diabetology 201312 21. (https://doi. org/10.1186/1475-2840-12-21)

19 Cibas ES \& Ali SZ. The 2017 Bethesda system for reporting thyroid cytopathology. Thyroid 201727 1341-1346. (https://doi.org/10.1089/ thy.2017.0500)

20 Meloun M, Hill M, Militky J \& Kupka K. Transformation in the PC-aided biochemical data analysis. Clinical Chemistry and Laboratory Medicine 200038 553-559. (https://doi.org/10.1515/ CCLM.2000.081)

21 Meloun M, Militky J, Hill M \& Brereton RG. Crucial problems in regression modelling and their solutions. Analyst 2002127 433-450. (https://doi.org/10.1039/b110779h)

22 Meloun M, Hill M, Militky J, Vrbikova J, Stanicka S \& Skrha J. New methodology of influential point detection in regression model

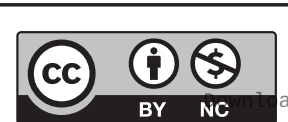

This work is licensed under a Creative Commons Attribution-NonCommercial 4.0 International License. ded from Bioscientifica,com at 04/26/2023 09:51:51AM 
building for the prediction of metabolic clearance rate of glucose. Clinical Chemistry and Laboratory Medicine 200442 311-322. (https:// doi.org/10.1515/CCLM.2004.057)

23 Trygg J, Holmes E \& Lundstedt T. Chemometrics in metabonomics. Journal of Proteome Research 20076 469-479. (https://doi. org/10.1021/pr060594q)

24 Trygg J \& Wold S. Orthogonal projections to latent structures (O-PLS). Journal of Chemometrics 200216 119-128. (https://doi. org/10.1002/cem.695)

25 Madsen R, Lundstedt T \& Trygg J. Chemometrics in metabolomics - a review in human disease diagnosis. Analytica Chimica Acta 2010 659 23-33. (https://doi.org/10.1016/j.aca.2009.11.042)

$26 \mathrm{Wu}$ P. Thyroid disease and diabetes. Clinical Diabetes 200018 365-372.

27 Malaguarnera R, Vella V, Nicolosi ML \& Belfiore A. Insulin resistance: any role in the changing epidemiology of thyroid cancer? Frontiers in Endocrinology 20178 314. (https://doi.org/10.3389/ fendo.2017.00314)

28 Mack WJ, Preston-Martin S, Bernstein L \& Qian D. Lifestyle and other risk factors for thyroid cancer in los Angeles County females. Annals of Epidemiology 200212 395-401. (https://doi.org/10.1016/ s1047-2797(01)00281-2)

29 Fleiner HF, Bjøro T, Midthjell K, Grill V \& Åsvold BO. Prevalence of thyroid dysfunction in autoimmune and type 2 diabetes: the population-based HUNT study in Norway. Journal of Clinical Endocrinology and Metabolism 2016101 669-677. (doi:10.1210/ jc.2015-3235)

30 Sotak Š, Lazurova I, Felsoci M, Novakova B \& Wagnerova H. The prevalence of type 2 diabetes mellitus in patients with autoimmune thyroiditis in hypothyroid stadium. Vnitrni Lekarstvi 201864 232-235.

31 Nishi M. Diabetes mellitus and thyroid diseases. Diabetology International 20189 108-112. (https://doi.org/10.1007/s13340-018$0352-4)$

32 Guo H, Sun M, He W, Chen H, Li W, Tang J, Tang W, Lu J, Bi Y, Ning G, et al. The prevalence of thyroid nodules and its relationship with metabolic parameters in a Chinese community-based population aged over 40 years. Endocrine 201445 230-235. (https:// doi.org/10.1007/s12020-013-9968-0)

33 Ayturk S, Gursoy A, Kut A, Anil C, Nar Demirer A \& Tutuncu NB. Metabolic syndrome and its components are associated with increased thyroid volume and nodule prevalence in a mild-tomoderate iodine deficient area. European Journal of Endocrinology 2009 161 599-605. (https://doi.org/10.1530/EJE-09-0410)

34 Boelaert K, Horacek J, Holder RL, Watkinson JC, Sheppard MC \& Franklyn JA. Serum thyrotropin concentration as a novel predictor of malignancy in thyroid nodules investigated by fine-needle aspiration. Journal of Clinical Endocrinology and Metabolism 200691 4295-4301. (https://doi.org/10.1210/jc.2006-0527)

35 Haymart MR, Repplinger DJ, Leverson GE, Elson DF, Sippel RS, Jaume JC \& Chen H. Higher serum thyroid stimulating hormone level in thyroid nodule patients is associated with greater risks of differentiated thyroid cancer and advanced tumor stage. Journal of Clinical Endocrinology and Metabolism 200893 809-814. (https://doi. org/10.1210/jc.2007-2215)

36 Fiore E, Rago T, Provenzale MA, Scutri M, Ugolinu C, Basolo F, Coscio Di G, Miccoli P, Grasso L, Pinchera A, et al. L-thyroxinetreated patients with nodular goiter have lower serum TSH and lower frequency of papillary thyroid cancer: results of a cross-sectional study on 27914 patients. Endocrine-Related Cancer 201017 231-239. (https://doi.org/10.1677/ERC-09-0251)

37 Duran AO, Anil C, Gursoy A, Nar A, Altundag O \& Tutuncu NB. The relationship between glucose metabolism disorders and malignant thyroid disease. International Journal of Clinical Oncology 201318 585-589. (https://doi.org/10.1007/s10147-012-0435-3)

38 Yalakanti D \& Dolia PB. Association of type II 5' monodeiodinase Thr92Ala single nucleotide gene polymorphism and circulating thyroid hormones among type 2 diabetes mellitus patients. Indian Journal of Clinical Biochemistry 201631 152-161. (https://doi. org/10.1007/s12291-015-0518-9)

39 Bagchi N. Thyroid function in a diabetic population. Special Topics in Endocrinology \& Metabolism 19823 45-55.

40 Shih SR, Chiu WY, Chang TC \& Tseng CH. Diabetes and thyroid cancer risk: literature review. Experimental Diabetes Research 2012 2012 578285. (https://doi.org/10.1155/2012/578285)

41 Chodick G, Heymann AD, Rosenmann L, Green MS, Flash S, Porath A, Kokia E \& Shalev V. Diabetes and risk of incident cancer: a large population-based cohort study in Israel. Cancer Causes \& Control 201021 879-887. (https://doi.org/10.1007/s10552-010-9515-8)

42 Aschebrook-Kilfoy B, Sabra MM, Brenner A, Moore SC, Ron E, Schatzkin A, Hollenbeck A \& Ward MH. Diabetes and thyroid cancer risk in the National Institutes of Health - AARP Diet and Health Study. Thyroid 201121 957-963. (https://doi.org/10.1089/ thy.2010.0396)

43 Luo J, Phillips L, Liu S, Wactawski-Wende J \& Margolis KL. Diabetes, diabetes treatment, and risk of thyroid cancer. Journal of Clinical Endocrinology and Metabolism 2016101 1243-1248. (https://doi. org/10.1210/jc.2015-3901)

44 Paes JE, Hua K, Nagy R, Kloos RT, Jarjoura D \& Ringel MD. The relationshipbetween body mass index and thyroid cancer pathology features and outcomes: aclinicopathological cohort study. Journal of Clinical Endocrinology and Metabolism 201095 4244-4250. (https:// doi.org/10.1210/jc.2010-0440)

45 Harikrishna A, Ishak A, Ellinides A, Saad R, Christodoulou H, Spartalis E \& Paschou SA. The impact of obesity and insulin resistance on thyroid cancer: a systematic review. Maturitas 2019125 45-49. (https://doi.org/10.1016/j.maturitas.2019.03.022)

46 World Health Organization. Diabetes Action Now: an initiative of the World Health Organization and the International Diabetes Federation. Geneva, Switzerland: World Health Organization, 2004 (available at: https://apps.who.int/iris/handle/10665/42934)

47 Beagley J, Guariguata L, Weil C \& Motala AA. Global estimates of undiagnosed diabetes in adults. Diabetes Research and Clinical Practice 2014103 150-160. (https://doi.org/10.1016/j.diabres.2013.11.001)

Received in final form 19 May 2020

Accepted 4 June 2020

Accepted Manuscript published online 4 June 2020 https://ec.bioscientifica.com https://doi.org/10.1530/EC-20-0180 (c) 2020 The authors Published by Bioscientifica Ltd

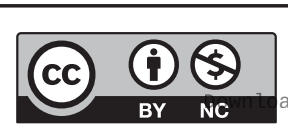

This work is licensed under a Creative Commons Attribution-NonCommercial 4.0 International License. ded from Bioscientifica.com at 04/26/2023 09:51:51AM 